\title{
Site fidelity, ontogenetic shift and diet composition of green turtles Chelonia mydas in Japan inferred from stable isotope analysis
}

\author{
Takahiro Shimada ${ }^{1,2, *}$, Shigeru Aoki ${ }^{1}$, Kazunari Kameda ${ }^{2}$, Julia Hazel ${ }^{3,4}$, \\ Kimberly Reich ${ }^{5}$, Naoki Kamezaki ${ }^{1,2,6}$ \\ ${ }^{1}$ Graduate School of Agricultural and Life Sciences, The University of Tokyo, Bunkyo-ku, Tokyo 113-8657, Japan \\ ${ }^{2}$ Sea Turtle Association of Japan, Hirakata, Osaka 573-0163, Japan \\ ${ }^{3}$ School of Earth and Environmental Sciences, James Cook University, Townsville, Queensland 4811, Australia \\ ${ }^{4}$ Centre for Tropical Water and Aquatic Ecosystem Research, Townsville, Queensland 4811, Australia \\ ${ }^{5}$ Department of Marine Biology, Texas A\&M University at Galveston, Galveston, Texas 77553, USA \\ ${ }^{6}$ Suma Aqualife Park, Kobe, Hyogo 654-0049, Japan
}

\begin{abstract}
Incomplete knowledge about local foraging ecology of green turtles hampers their conservation management in Japan, where stocks have only partially recovered from heavy exploitation in previous centuries. We used stable isotope ratios of $\delta^{13} \mathrm{C}$ and $\delta^{15} \mathrm{~N}$ for turtle carapace scutes, where successive layers contain a chronological record of diet assimilated over a period of years. Turtles were sampled at 2 geographically separate foraging grounds in Japan: the temperate Main Islands $(\mathrm{n}=32)$ and the sub-tropical Nansei Islands $(\mathrm{n}=42)$. Site fidelity was inferred for the majority of turtles at each site (81 and $64 \%$ resident turtles) because isotope data indicated diets consistent with food taxa at the respective sites. Immigrant turtles (previous diet outside their current site) were few $(\mathrm{n}=4)$ at the Main Islands site but numerous $(\mathrm{n}=14)$ at the Nansei Islands site, where they were significantly smaller than residents. An ontogenetic shift (Main Islands to Nansei Islands) was inferred for many of the immigrants on the basis of isotope evidence and body size. These immigrants corresponded to a size cohort that was relatively scarce in Main Islands foraging grounds according to previous studies. Bayesian mixing models, used to estimate proportional components of diet, showed varying degrees of imbalance between seagrass and algae and indicated that hypothetical consumption of non-trivial amounts of animal matter was plausible. The latter represented a hypothetical diet component for study turtles since animal matter was rarely found in stomach contents. Potential ambiguity and other issues that constrained inference from mixing models are discussed.
\end{abstract}

KEY WORDS: Green Turtle $\cdot$ Foraging site fidelity $\cdot$ Ontogenetic shift $\cdot$ Diet composition $\cdot$ Stable isotope analysis $\cdot \delta^{13} \mathrm{C} \cdot \delta^{15} \mathrm{~N} \cdot$ Chelonia mydas

- Resale or republication not permitted without written consent of the publisher

\section{INTRODUCTION}

Green turtles Chelonia mydas are vulnerable or endangered throughout their range (Seminoff 2004, Wallace et al. 2011), and their global decline is largely due to anthropogenic activities (Jackson et al. 2001, Frazier 2003). In Japan, the Ministry of Environment
(MOE) lists green turtles as a vulnerable species (MOE 2012), but stocks were heavily exploited in previous centuries, particularly in Ogasawara during the 1800s. Up to 3000 turtles were harvested per year in peak periods, but the annual catch had declined to approximately 100 individuals in 1930 (Sato 1989). Despite recent increases in the Ogasawara nesting 
population, the Japanese stock remains below historical abundance and requires continuous conservation management, as is the case for depleted stocks in other parts of the world (Suganuma 1998, Chaloupka et al. 2008, Kittinger et al. 2013).

Understanding the foraging ecology of green turtles is essential for the development of successful conservation and management programmes (Bjorndal 1999, Martin et al. 2007, Hamann et al. 2010), but the task is challenging because both movement and diet of the species vary between ontogenetic stages. Initially, green turtle hatchlings migrate from their natal beaches to oceanic habitats where evidence suggests they drift passively with ocean currents and consume an omnivorous diet (Balazs 1976, Wyneken \& Salmon 1992, Boyle \& Limpus 2008, Mansfield et al. 2014). After several years, small juveniles enter neritic habitats (Bjorndal 1997, Reich et al. 2007, Arthur et al. 2008) where, in general, green turtles spend the major part of their lives and consume a diet that is predominantly, but not exclusively, herbivorous (Bjorndal 1997). After reaching maturity, most green turtles migrate at irregular intervals between their foraging grounds and distant nesting sites, with female turtles exhibiting high site fidelity over many years (Limpus et al. 1992, Balazs 1994, Miller 1997).

The post-oceanic ecology of green turtles appears to vary widely among geographic regions. For example, in the western North Atlantic, juvenile green turtles are understood to shift from temperate and subtropical habitats to the tropical Caribbean (the natal region for many of them) based on studies of size distribution (e.g. Mendonca \& Ehrhart 1982, Bjorndal \& Bolten 1988, Epperly et al. 1995, Meylan et al. 2011), mark-recapture (Bjorndal \& Bolten 1995, Moncada et al. 2006, Meylan et al. 2011) and genetic analysis (Lahanas et al. 1998, Bass \& Witzell 2000). In the western South Atlantic, some juvenile green turtles make seasonal shifts between neritic and oceanic habitats, as revealed by satellite telemetry (González Carman et al. 2012, 2014). However, similar shifts have not been reported for green turtles in the western Pacific, and long-term fidelity to neritic foraging sites has been confirmed in eastern Australia by markrecapture studies (Limpus et al. 1994, Chaloupka et al. 2004).

In Japan, green turtle breeding sites are restricted to the Ogasawara and Nansei Islands (Kamezaki 1989, Yamaguchi et al. 2005), while foraging grounds exist both in these remote island groups and along the coast of the Main Islands (Uchida \& Nishiwaki 1982, Ogasawara Marine Center 1994, Shimada 2008) (Fig. 1). Natal origins of adult and juvenile green tur- tles in Japan have been elucidated through markrecapture, satellite telemetry and genetic analysis. Turtles foraging at Main Islands sites originated predominantly from the Ogasawara Islands, with limited records from Taiwan and Micronesia (Tachikawa \& Sasaki 1990, Ogasawara Marine Center 1994, Cheng 2000, Miyawaki et al. 2000, Hatase et al. 2006, Nishizawa et al. 2013). Turtles foraging in the Nansei Islands had diverse origins in the western Pacific and Indian Oceans (Ogasawara Marine Center 1994, Cheng 2000, Song et al. 2002, Hamabata et al. 2009, Nishizawa et al. 2013). In contrast to detailed scientific insight regarding natal origins, fidelity to foraging sites, possible ontogenetic shifts and variation in diet remain obscure for Japanese green turtles. Our study addresses this knowledge gap.

Green turtle foraging aggregations in the Main Islands and the Nansei Islands encompass the size range from small juveniles (straight carapace length [SCL] $\sim 40 \mathrm{~cm}$ ) to adults $(\mathrm{SCL}>82 \mathrm{~cm})($ e.g. Okamoto et al. 2011 and references therein), but measurements reported in earlier studies imply a disparity in size distribution. Juvenile turtles of intermediate size (approximate SCL range: 50 to $70 \mathrm{~cm}$ ) were notably under-represented at Main Islands sites (e.g. Okamoto et al. 2011 and references therein), whereas turtles in this size range were relatively abundant at southern Nansei Islands sites (Kameda et al. 2013).

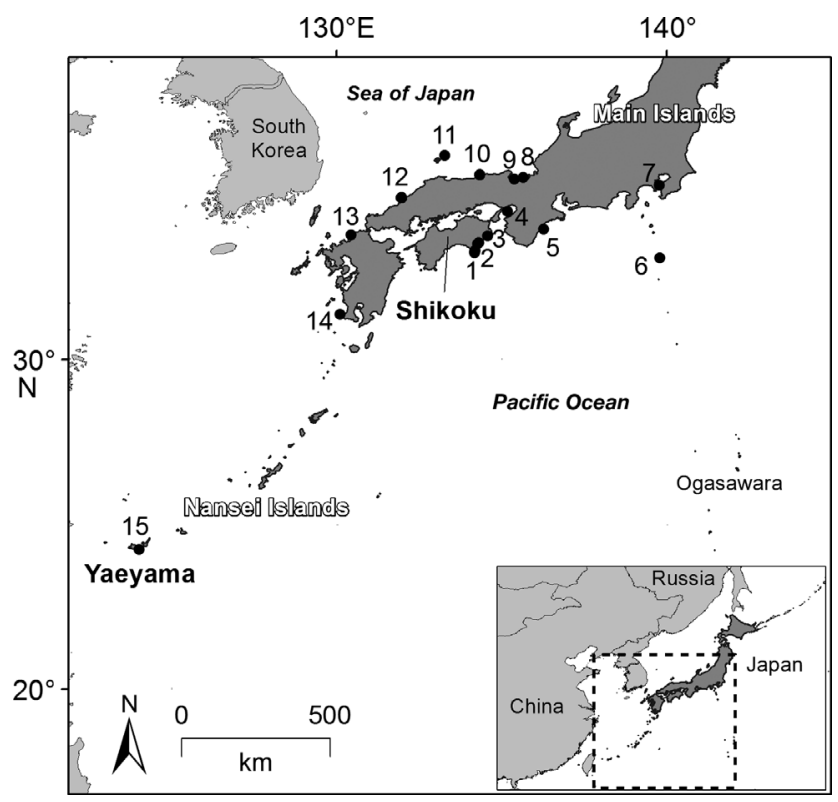

Fig. 1. Chelonia mydas. Green turtles were sampled at 2 geographically separate foraging grounds in Japan: southeastern Shikoku and Yaeyama. Turtle food sources were sampled at 15 locations $(\bullet)$. Dashed-line rectangle in the regional map (inset, lower right) shows the geographic extent of the detailed map 
We surmised that the under-represented size cohort at Main Islands foraging grounds could indicate an ontogenetic shift by juvenile turtles of intermediate size moving away from the Main Islands, with the southern Nansei Islands a plausible successive foraging habitat.

As a tool for investigating residency at these 2 geographically separate foraging grounds and possible movement between them, we turned to stable isotope analysis. The rationale underlying this method is well established: stable isotope ratios in animal tissue reflect the stable isotope ratios of their food sources (Fry 2006). Correspondence of isotope ratios between animal tissue and known food sources at a locality can be used to infer residence. Similarly, movement can be inferred when tissue isotopes differ from those of local food sources. In addition, mathematical mixing models can be used to estimate the proportions of various food sources that have contributed to tissue formation (Fry 2006).

In our study, mixing models would allow us to estimate major components of green turtle diets from the isotope ratios of their tissues. Prior studies have found gut contents of Main Islands turtles were dominated by macroalgae, whereas both macroalgae and seagrass were found in the gut contents of turtles at the Nansei Islands (Kameda \& Ishihara 2009, Suganuma et al. 2010). It has been inferred that each individual turtle forages preferentially either on macroalgae or on seagrass (Sea Turtle Association of Japan, STAJ, unpubl. data), but this understanding is based on a 'snapshot' view of diet provided by gut contents. Insight regarding the relative importance of assimilated macroalgae and seagrass might be gained from stable isotopes that reflect diet over a longer period.

In addition, we wanted to investigate the possible consumption of animal matter by green turtles in both foraging regions of Japan. Prior studies using gut contents analysis have reported only a minor component of animal matter, e.g. $<5.5 \%$ animal matter by volume (Kameda \& Ishihara 2009). However, inference from the stable isotope ratios of green turtle eggs suggested that some nesting females in the Ogasawara Islands consumed a substantial proportion of invertebrates prior to breeding migration (Hatase et al. 2006). Consumption of animal matter by green turtles in other parts of the world has been confirmed by direct observation of episodic ingestion or inferred from stable isotope data or both (Heithaus et al. 2002, Seminoff et al. 2006b, Amorocho \& Reina 2007, Arthur et al. 2007, Cardona et al. 2010, Burkholder et al. 2011, Lemons et al. 2011).
In summary, our primary objective was to measure stable isotope ratios of green turtle scute tissue, compare the tissue data with stable isotope ratios of local food sources and, hence, infer either residence in the capture area or immigration from a different area. In addition, we wanted to use mixing models to estimate (a) the relative contributions of seagrass and macroalgae and (b) the hypothetical inclusion of animal matter in the diets of study turtles.

\section{MATERIALS AND METHODS}

To allow comparison between 2 geographically separate green turtle foraging regions in Japan, our study sites were located (1) at south-eastern Shikoku (Shikoku hereafter) in the Main Islands and (2) at Yaeyama in the Nansei Islands group (Fig. 1). Green turtles were sampled between May 2010 and November 2011.

\section{Tissue and food samples}

Study turtles comprised stranded animals, turtles accidentally caught in coastal fisheries (5 to $78 \mathrm{~m}$ depth), and turtles caught for research using entanglement nets (stretched mesh size $=45 \mathrm{~cm}$, water depth $\leq 2 \mathrm{~m}$ ). SCL was measured between the nuchal notch and posterior tip of the carapace (Uchida 1967). Turtles with SCL > $82 \mathrm{~cm}$ were considered to be adult size, following data for nesting turtles (Tachikawa 1991).

Turtle tissue was sampled from the carapace because chronological changes in diet can be detected by measuring the isotope ratios of successive layers of carapace scutes (Reich et al. 2007). We sampled carapace tissue using a sterile cutter to remove approximately $1 \mathrm{~cm}^{2}$ from anterior and posterior sites on the second costal scute (Fig. 2) according to the method of Reich et al. (2007) and based on the pattern of scute formation (Kobayashi 2001). For a small subset of turtles we also obtained skin tissue samples (approximately $1 \mathrm{~cm}^{2}$ ) from the shoulder area. The latter were used to derive an adjustment to discrimination values as described in the Supplement at www.int-res.com/articles/suppl/n025p151_ supp.pdf.

We assembled food samples from multiple sources. Field samples were collected at coastal sites up to $3 \mathrm{~m}$ depth (Fig. 1: Locations 1-3, 7, 9, 15). We collected only those taxa known to be eaten by green turtles in Japan, based on identification of stomach contents in 


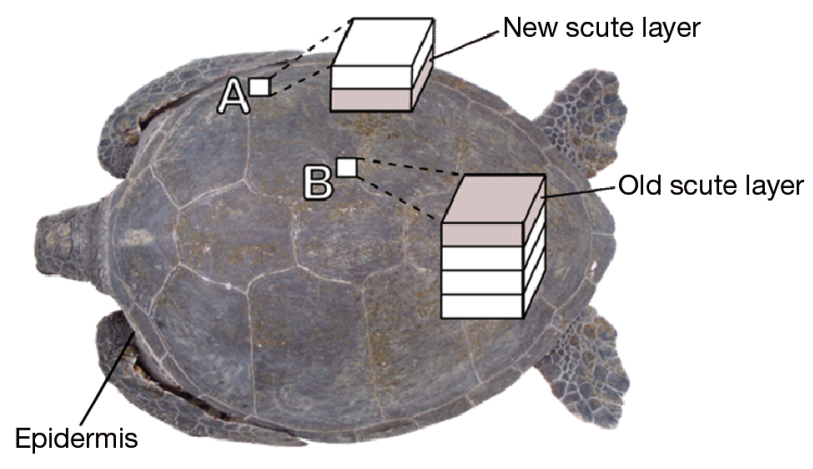

Fig. 2. Chelonia mydas. Carapace tissue was sampled at anterior (A) and posterior (B) positions on the second costal scute. Stable isotope ratios were measured for newly synthesized tissue from the basal layer at $\mathrm{A}$ and the oldest retained tissue from the surface layer at $B$, in order to infer the recent and previous diet of the turtle. Skin tissue (epidermis) was sampled at the shoulder area. Figure adapted from Reich et al. (2007)

prior research (Kameda \& Ishihara 2009). Ingested food samples were obtained from live turtles by stomach lavage (Forbes \& Limpus 1993) and from freshly dead turtles by dissection. Ingested items were identified to the lowest possible taxonomic level using standard keys (Yoshida 1998). Macroalgal and seagrass samples were subsequently prepared for stable isotope analysis as detailed in the next section. Animal matter in stomach contents was not used in analysis because it was found only in rare instances, and because animal matter may be altered in the stomachs of some animals (Hwang et al. 2007). Digestion of plant cell walls occurs in the hindgut of green turtles (Bjorndal et al. 1991). Therefore, isotope ratios of macroalgae and seagrass were not expected to be altered in turtle stomachs. To validate this expectation, stable isotope ratios of stomach samples and field samples were compared statistically. Finally, we obtained data from prior studies for potential food sources (Takai et al. 2001, Hatase et al. 2002, 2006).

\section{Stable isotope samples}

All samples were stored frozen $\left(-18^{\circ} \mathrm{C}\right)$ prior to preparation. Surface contamination was removed by careful scrubbing and rinsing in distilled water. A portion of each sample was tested in hydrochloric acid solution (1 N HCL; Longin 1971). Absence of bubbles confirmed absence of calcium carbonates in every case. Therefore, the remaining portions were deemed suitable for stable isotope analysis without pre-treatment. Acid-treated portions were not used in analysis.

Scute material was mounted horizontally on a wooden block with epoxy glue to facilitate the removal of very thin layers $(50 \mu \mathrm{m})$ with a microtome. From each scute we obtained separate samples of (1) recently synthesized tissue from the basal layer of the anterior site (A in Fig. 2), hereafter termed 'new' scute tissue, from which 'recent' diet was inferred, and (2) 'old' tissue from the surface layer of the posterior site (B in Fig. 2), from which 'previous' diet was inferred. All samples were pulverized in a mill homogenizer, treated for lipid extraction with a 2:1 mixture of chloroform and methanol (Folch et al. 1957) and dried at $60^{\circ} \mathrm{C}$ for $24 \mathrm{~h}$.

Elemental analysis provided carbon and nitrogen isotope ratios $\delta^{13} \mathrm{C}$ and $\delta^{15} \mathrm{~N}$, reported as relative deviation (\%o) from the conventional standards PeeDee Belemnite and atmospheric nitrogen, respectively (Fry 2006). Laboratory-calibrated alanine samples were inserted after every 10 research samples for control. Standard deviation of the alanine samples was below $\pm 0.2 \%$ for $\delta^{13} \mathrm{C}$ and $\delta^{15} \mathrm{~N}$, confirming the precision of analysis. For all analyses the $\delta^{13} \mathrm{C}$ and $\delta^{15} \mathrm{~N}$ data were corrected for diet-tissue discrimination. We estimated discrimination values for scute tissue by applying an empirically derived adjustment to published discrimination values for skin tissue (Seminoff et al. 2006a). Details of the adjustment are available in (Table S1 in the Supplement at www.int-res.com/articles/suppl/n025p151_ supp.pdf).

\section{Stable isotope analysis}

The first stage of our analysis considered only confirmed food sources (taxa identified in samples of ingested material from green turtles in Japan). These were represented by samples collected at our study sites, Shikoku and Yaeyama. Hereafter we refer to these samples collectively as 'known food'. The isotopic boundary of known food was defined for each study site by a minimum convex polygon enclosing the isotope ratios $\left(\delta^{13} \mathrm{C}\right.$ and $\left.\delta^{15} \mathrm{~N}\right)$ of food samples with a buffer of width equal to the precision of our elemental analysis $(0.2 \%)$. We then evaluated scute data for each turtle in relation to the boundary at the relevant capture site. When a scute sample had isotope ratios within the boundary, a diet consistent with known food at the site was inferred. Conversely, when a scute sample had isotope ratios beyond the boundary, a diet sourced outside the site was inferred. 
Study turtles were then classified according to their inferred diet as follows: (1) If both new and old scute samples indicated a diet consistent with known food at the site, the turtle was classified as 'resident'. (2) If the old scute sample indicated a diet obtained outside the site, the turtle was classified as 'immigrant'. For the latter, the new scute could fall inside or outside the boundary, allowing for diverse durations since recruitment to the study site. (3) If the old scute indicated diet within the site and the new scute indicated diet outside the site, the turtle was classified as 'nomad'.

In the next stage of analysis, we evaluated the relative importance of macroalgae and seagrass for Yaeyama resident turtles through the use of mixing models as detailed below. Food sources used in this analysis were the known food for Yaeyama. This analysis excluded other turtles because seagrass was rare at Shikoku and food availability was unknown for immigrants and nomads.

Finally, we considered the potential dietary contribution of animal food such as gelatinous and softbodied invertebrates, although such items were rarely identified in ingested samples. In addition to the data for known food, we needed proxy data to represent this hypothetical diet component. We used isotope ratios for macroplanktonic invertebrates from prior studies in which these invertebrates represented potential prey of chelonians in Japanese waters (Hatase et al. 2002, 2006). These data for invertebrates were the most appropriate proxy data available for our purpose, although the samples had been obtained at a location distant from our study sites. The sampling area was off the Sanriku coast of the Main Islands of Japan (Hatase et al. 2002, 2006).

We ran Bayesian mixing models for diet source contribution in SIAR (Stable Isotope Analysis in $\mathrm{R}_{\text {; }}$ Parnell \& Jackson 2013). For each stage of our mixing analysis, the SIAR model, fit via Markov Chain Monte Carlo, was run for 500000 iterations with a burn-in of 50000 . The model produced probability density distributions of potential dietary solutions (Parnell et al. 2010). We confirmed uni-modality of the distributions and thus used the median values to estimate the most probable contribution of each food source to the diet of each turtle (Moore \& Semmens 2008). The 2-sample $t$-test was used for parametric data, and the Wilcoxon rank-sum test was used for non-parametric data. Shapiro-Wilk test and Levene's test were used to examine normality and homogeneity of variance. All statistical analyses were conducted using R software (R Core Team 2013).

\section{RESULTS}

We sampled a total of 74 green turtles comprising 32 turtles at Shikoku (SCL $=37.6$ to $91.3 \mathrm{~cm}$, median $=45.5 \mathrm{~cm}$ ) and 42 turtles at Yaeyama $(\mathrm{SCL}=42.5$ to $83.0 \mathrm{~cm}$, median $=53.5 \mathrm{~cm}$ ). For each individual we obtained $\delta^{13} \mathrm{C}$ and $\delta^{15} \mathrm{~N}$ values for new and old scute tissue (Table 1). We compiled $\delta^{13} \mathrm{C}$ and $\delta^{15} \mathrm{~N}$ values of green turtle food sources sampled at our 2 study sites and at other Main Island sites (Tables $2 \& 3$ ). Use of stable isotope values from ingested samples (from stomach contents) was validated by $t$-tests that showed no significant difference between stomach and field samples of the dominant species Gelidium elegans $\left(\delta^{13} \mathrm{C}\right.$ values $t_{(2) 10}=-1.23, \mathrm{p}=0.25 ; \delta^{15} \mathrm{~N}$ values $t_{(2) 10}=1.04, \mathrm{p}=0.32$ ).

New scute layers of Shikoku turtles were more depleted in ${ }^{13} \mathrm{C}$ and enriched in ${ }^{15} \mathrm{~N}$ as compared with the corresponding measures for turtles at Yaeyama (Fig. 3, Table 1). Differences were statistically significant for $\delta^{13} \mathrm{C}$ values $(W=19, \mathrm{p}<0.0001)$ and for $\delta^{15} \mathrm{~N}$ values $\left(t_{(2) 72}=9.90, \mathrm{p}<0.0001\right)$, indicating diets differentiated by site. The majority of turtles were classified as resident at their respective sites, by inference from stable isotope ratios indicating previous and recent diet consistent with known food for the site: Shikoku, $\mathrm{n}=26$ (81\%); Yaeyama, $\mathrm{n}=27$ (64\%) (Fig. 3, Table 1).

Immigrants (previous diet outside the site) were numerous at Yaeyama $(\mathrm{n}=14,33 \%)$ and were significantly smaller than residents $(W=290.5, \mathrm{p}<0.01)$. In contrast, Shikoku had few immigrants ( $\mathrm{n}=4,13 \%$ ), and these were not significantly different in size to residents $(W=55.5, \mathrm{p}=0.85)$. Nomads (Shikoku, $\mathrm{n}=2$; Yaeyama, $\mathrm{n}=1$ ) were too few for statistical evaluation and were excluded from further analysis (Fig. 3, Table 1).

The relative importance of seagrass and macroalgae was assessed for Yaeyama residents, the only study turtles for which both food sources could be assumed continuously available. Mixing model results are summarized here as old\%lnew\% (based on isotope data for old and new scutes) to indicate estimates for the proportion of a food source in the previous and recent diet, respectively. Macroalgae appeared to be more important for 5 turtles $(85 \% 187 \%$; $80 \% \mid 81 \%$; $80 \% \mid 71 \%$; $93 \% \mid 76 \%$; $83 \% \mid 62 \%)$, while seagrass appeared more important for 3 turtles (88\%|90\%; 86\%|82\%: $76 \% \mid 81 \%$ ). For the remainder, estimates were lower and varied. For 14 turtles both estimates (previous and recent) were $>50 \%$ macroalgae, while for 3 turtles both estimates were $>50 \%$ seagrass. Two turtles had an estimated previous diet 
Table 1. Chelonia mydas. Stable isotope ratios $\left(\delta^{13} \mathrm{C}, \delta^{15} \mathrm{~N}\right)$ of green turtles sampled at 2 study sites, Shikoku and Yaeyama. SCL: straight carapace length. Status was inferred from stable isotope data-R: resident; I: immigrant; N: nomad

\begin{tabular}{|c|c|c|c|c|c|c|c|c|c|c|c|c|c|}
\hline \multirow{2}{*}{$\begin{array}{l}\text { Turtle } \\
\text { ID }\end{array}$} & \multirow{2}{*}{$\begin{array}{l}\mathrm{SCL} \\
(\mathrm{cm})\end{array}$} & \multicolumn{2}{|c|}{ New scute layer } & \multicolumn{2}{|c|}{ Old scute layer } & \multirow{2}{*}{$\begin{array}{c}\text { Inferred } \\
\text { status }\end{array}$} & \multirow{2}{*}{$\begin{array}{l}\text { Turtle } \\
\text { ID }\end{array}$} & \multirow{2}{*}{$\begin{array}{l}\text { SCL } \\
(\mathrm{cm})\end{array}$} & \multicolumn{2}{|c|}{ New scute layer } & \multicolumn{2}{|c|}{ Old scute layer } & \multirow{2}{*}{$\begin{array}{c}\text { Inferred } \\
\text { status }\end{array}$} \\
\hline & & $\delta^{13} \mathrm{C}(\%)$ & $\delta^{15} \mathrm{~N}(\%)$ & $\delta^{13} \mathrm{C}(\%)$ & $\delta^{15} \mathrm{~N}(\%)$ & & & & $\delta^{13} \mathrm{C}(\% \circ)$ & $\delta^{15} \mathrm{~N}(\%$ \%) & $\delta^{13} \mathrm{C}(\%)$ & $\delta^{15} \mathrm{~N}(\%)$ & \\
\hline \multicolumn{7}{|c|}{ Shikoku } & Y05 & 43.3 & -14.8 & 7.4 & -15.3 & 6.1 & $\mathrm{R}$ \\
\hline S01 & 37.6 & -17.6 & 9.1 & -18.1 & 6.9 & $\mathrm{R}$ & Y06 & 43.7 & -15.3 & 7.4 & -18.5 & 6.0 & $\mathrm{R}$ \\
\hline S02 & 37.7 & -17.5 & 9.8 & -18.3 & 5.0 & I & Y07 & 44.6 & -16.0 & 8.8 & -18.7 & 6.1 & $\mathrm{~N}$ \\
\hline S03 & 39.3 & -17.7 & 9.2 & -20.0 & 7.9 & $\mathrm{R}$ & Y08 & 44.8 & -15.3 & 7.3 & -17.6 & 7.2 & I \\
\hline S04 & 39.3 & -18.5 & 7.1 & -19.8 & 7.4 & $\mathrm{R}$ & Y09 & 45.6 & -12.7 & 7.1 & -16.4 & 11.0 & I \\
\hline S05 & 39.4 & -18.6 & 9.5 & -19.4 & 9.1 & $\mathrm{R}$ & Y10 & 46.4 & -9.9 & 4.1 & -9.1 & 4.4 & $\mathrm{R}$ \\
\hline S06 & 40.3 & -19.1 & 9.0 & -19.3 & 8.5 & $\mathrm{R}$ & Y11 & 47.8 & -14.1 & 7.0 & -18.9 & 10.0 & I \\
\hline S07 & 40.6 & -18.6 & 9.3 & -18.7 & 8.0 & $\mathrm{R}$ & Y12 & 48.1 & -14.2 & 7.8 & -15.4 & 7.7 & $\mathrm{R}$ \\
\hline S08 & 40.6 & -17.8 & 8.4 & -17.6 & 9.3 & $\mathrm{R}$ & Y13 & 49.0 & -14.4 & 6.6 & -15.0 & 6.2 & $\mathrm{R}$ \\
\hline S09 & 40.9 & -18.4 & 8.7 & -19.6 & 7.8 & $\mathrm{R}$ & Y14 & 49.3 & -14.0 & 7.5 & -14.2 & 8.3 & I \\
\hline S10 & 41.1 & -18.5 & 7.8 & -19.3 & 8.6 & $\mathrm{R}$ & $\begin{array}{l}114 \\
\text { Y15 }\end{array}$ & 50.2 & -13.2 & 6.2 & -16.6 & 13.9 & I \\
\hline S11 & 41.6 & -19.0 & 8.5 & -19.3 & 7.7 & $\mathrm{R}$ & Y16 & 50.6 & -13.4 & 5.4 & -13.2 & 6.1 & $\mathrm{R}$ \\
\hline S12 & 42.0 & -18.7 & 9.4 & -18.1 & 5.8 & I & Y17 & 51.3 & -14.3 & 6.1 & -15.5 & 9.2 & I \\
\hline S13 & 42.0 & -18.5 & 8.0 & -18.3 & 6.2 & $\mathrm{R}$ & $\begin{array}{l}\mathrm{Y} 1 \mathrm{t} \\
\mathrm{Y} 18\end{array}$ & 51.6 & -11.4 & 6.1 & -13.1 & 6.7 & $\mathrm{R}$ \\
\hline S14 & 42.4 & -18.4 & 8.6 & -19.3 & 8.4 & $\mathrm{R}$ & Y19 & 52.0 & -14.1 & 6.4 & -19.3 & 8.4 & I \\
\hline S15 & 42.5 & -19.1 & 8.1 & -19.7 & 9.6 & $\mathrm{R}$ & Y20 & 52.6 & -13.5 & 5.5 & -15.7 & 6.4 & $\mathrm{R}$ \\
\hline S16 & 43.6 & -19.1 & 8.4 & -18.9 & 7.8 & $\mathrm{R}$ & Y21 & 52.6 & -16.5 & 6.9 & -16.3 & 7.4 & $\mathrm{R}$ \\
\hline S17 & 47.4 & -19.3 & 9.4 & -19.4 & 8.6 & $\mathrm{R}$ & Y22 & 54.3 & -14.7 & 7.3 & -14.1 & 6.5 & $\mathrm{R}$ \\
\hline S18 & 50.8 & -18.9 & 9.2 & -19.1 & 10.0 & $\mathrm{R}$ & Y23 & 54.4 & -10.7 & 5.1 & -12.4 & 5.2 & $\mathrm{R}$ \\
\hline S19 & 59.1 & -17.8 & 10.9 & -18.4 & 10.6 & $\mathrm{R}$ & Y24 & 55.5 & -13.3 & 5.9 & -13.5 & 5.9 & $\mathrm{R}$ \\
\hline S20 & 63.3 & -18.3 & 10.5 & -17.6 & 12.1 & I & Y25 & 55.8 & -14.4 & 7.0 & -13.8 & 7.1 & $\mathrm{R}$ \\
\hline S21 & 64.3 & -18.2 & 9.2 & -17.5 & 10.0 & $\mathrm{R}$ & Y26 & 56.1 & -15.0 & 6.9 & -15.6 & 8.9 & I \\
\hline S22 & 68.6 & -16.3 & 8.3 & -16.0 & 8.5 & $\mathrm{R}$ & Y27 & 56.5 & -15.5 & 6.7 & -15.7 & 7.3 & $\mathrm{R}$ \\
\hline $\mathrm{S} 23$ & 74.7 & -17.1 & 7.7 & -16.9 & 7.5 & $\mathrm{R}$ & $\begin{array}{l}\mathrm{Y} 27 \\
\mathrm{Y} 28\end{array}$ & $\begin{array}{l}30.5 \\
57.2\end{array}$ & -9.8 & 4.1 & -13.8 & 6.1 & $\mathrm{R}$ \\
\hline S24 & 75.9 & -22.2 & 7.7 & -23.5 & 8.0 & I & Y29 & 57.4 & -13.5 & 5.8 & -14.7 & 5.2 & $\mathrm{R}$ \\
\hline S25 & 77.6 & -19.0 & 9.5 & -18.6 & 10.2 & $\mathrm{R}$ & Y30 & 57.7 & -8.4 & 4.4 & -8.9 & 4.4 & $\mathrm{R}$ \\
\hline S26 & 77.7 & -18.4 & 7.7 & -17.7 & 9.1 & $\mathrm{R}$ & Y31 & 58.6 & -18.3 & 5.0 & -25.8 & 5.1 & I \\
\hline S27 & 78.7 & -19.3 & 8.9 & -18.0 & 9.0 & $\mathrm{R}$ & Y32 & 59.9 & -12.8 & 6.7 & -13.8 & 6.7 & $\mathrm{R}$ \\
\hline S28 & 81.7 & -17.4 & 11.7 & -16.3 & 10.4 & $\mathrm{~N}$ & Y33 & 61.3 & -14.5 & 7.2 & -13.5 & 6.8 & $\mathrm{R}$ \\
\hline S29 & 88.0 & -17.8 & 8.8 & -17.6 & 9.2 & $\mathrm{R}$ & Y34 & 62.7 & -12.1 & 6.1 & -13.0 & 6.6 & $\mathrm{R}$ \\
\hline S30 & 89.1 & -16.1 & 9.2 & -16.5 & 8.6 & $\mathrm{R}$ & Y35 & 62.9 & -9.5 & 5.3 & -11.2 & 5.8 & $\mathrm{R}$ \\
\hline S31 & 90.3 & -18.3 & 12.2 & -19.0 & 11.2 & $\mathrm{~N}$ & Y36 & 63.9 & -16.5 & 5.8 & -13.3 & 4.8 & $\mathrm{R}$ \\
\hline S32 & 91.3 & -17.1 & 10.4 & -17.5 & 9.6 & $\mathrm{R}$ & Y37 & 67.0 & -9.1 & 5.5 & -9.7 & 5.2 & $\mathrm{R}$ \\
\hline \multicolumn{7}{|c|}{ Yaeyama } & Y38 & 70.5 & -8.4 & 6.1 & -11.1 & 7.0 & $\mathrm{R}$ \\
\hline Y01 & 42.5 & -13.9 & 8.7 & -19.8 & 8.1 & I & Y39 & 71.3 & -15.0 & 7.5 & -13.2 & 6.5 & $\mathrm{R}$ \\
\hline Y02 & 42.7 & -14.5 & 7.0 & -18.7 & 7.6 & I & Y40 & 72.5 & -15.1 & 7.7 & -13.4 & 7.8 & $\mathrm{R}$ \\
\hline Y03 & 42.9 & -16.4 & 6.1 & -18.3 & 10.6 & I & Y41 & 73.2 & -13.3 & 7.0 & -15.2 & 7.8 & $\mathrm{R}$ \\
\hline Y04 & 43.3 & -15.3 & 7.2 & -18.9 & 6.9 & I & Y42 & 83.0 & -7.6 & 6.8 & -6.3 & 5.8 & I \\
\hline
\end{tabular}

Table 2. Stable isotope ratios $\left(\delta^{13} \mathrm{C}, \delta^{15} \mathrm{~N}\right)$ of food source taxa sampled from field sites and turtle stomachs. Means ( \pm SD) provided where multiple samples were available. $\mathrm{n}$ : number of samples; SCL: straight carapace length. Location numbers correspond to sampling sites marked in Fig. 1

\begin{tabular}{|c|c|c|c|c|c|c|}
\hline Taxon & $\mathrm{n}$ & $\delta^{13} \mathrm{C}(\% \circ)$ & $\delta^{15} \mathrm{~N}(\%)$ & Location & Sampling method & $\mathrm{SCL}(\mathrm{cm})$ \\
\hline \multicolumn{7}{|l|}{ Shikoku macroalgae ${ }^{a}$} \\
\hline Gelidium elegans & 1 & -16.9 & 6.4 & 1. Muroto & Stomach & 43.3 \\
\hline Gelidium elegans & 1 & -16.9 & 6.5 & 1. Muroto & Stomach & 54.3 \\
\hline Gelidium elegans & 1 & -14.5 & 5.7 & 1. Muroto & Stomach & 39.3 \\
\hline Unidentified species & 1 & -22.0 & 7.8 & 1. Muroto & Stomach & 43.6 \\
\hline Gelidium elegans & 1 & -15.4 & 5.5 & 1. Muroto & Field & - \\
\hline Codium sp. & 1 & -17.6 & 4.0 & 1. Muroto & Stomach & 74.7 \\
\hline Halymeniaceae & 1 & -17.6 & 4.2 & 1. Muroto & Stomach & 74.7 \\
\hline Gelidium elegans & 1 & -17.5 & 6.0 & 1. Muroto & Stomach & 74.7 \\
\hline Gelidium elegans & 1 & -17.4 & 6.0 & 1. Muroto & Stomach & 47.4 \\
\hline
\end{tabular}


Table 2 (continued)

\begin{tabular}{|c|c|c|c|c|c|c|}
\hline Taxon & $\mathrm{n}$ & $\delta^{13} \mathrm{C}(\%)$ & $\delta^{15} \mathrm{~N}(\%)$ & Location & Sampling method & $\mathrm{SCL}(\mathrm{cm})$ \\
\hline Halymeniaceae & 1 & -13.5 & 8.1 & 1. Muroto & Stomach & 47.4 \\
\hline Prionitis angusta & 1 & -13.0 & 8.3 & 1. Muroto & Stomach & 47.4 \\
\hline Meristotheca sp. & 1 & -21.6 & 4.5 & 1. Muroto & Field & - \\
\hline Gelidium elegans & 1 & -15.3 & 5.5 & 1. Muroto & Field & - \\
\hline Codium intricatum & 1 & -12.9 & 5.6 & 1. Muroto & Field & - \\
\hline Gigartinales & 1 & -17.2 & 7.4 & 1. Muroto & Stomach & 40.9 \\
\hline Gelidium elegans & 1 & -16.5 & 6.5 & 1. Muroto & Stomach & 40.9 \\
\hline Codium sp. & 1 & -15.7 & 7.5 & 1. Muroto & Stomach & 40.9 \\
\hline Gelidium sp. & 1 & -14.6 & 6.2 & 1. Muroto & Stomach & 40.9 \\
\hline Gelidium sp. & 1 & -18.5 & 5.5 & 1. Muroto & Stomach & 68.6 \\
\hline Halymeniaceae & 1 & -17.1 & 4.2 & 1. Muroto & Stomach & 68.6 \\
\hline Unidentified species & 1 & -16.0 & 8.0 & 1. Muroto & Stomach & 81.7 \\
\hline Unidentified species & 1 & -15.2 & 7.2 & 1. Muroto & Stomach & 81.7 \\
\hline Gelidium elegans & 1 & -16.8 & 7.2 & 1. Muroto & Field & - \\
\hline Gelidium elegans & 1 & -16.1 & 6.9 & 1. Muroto & Field & - \\
\hline Gelidium sp. & 1 & -18.8 & 6.0 & 2. Shishikui-ikumi & Stomach & 37.3 \\
\hline Gelidium elegans & 1 & -17.4 & 6.2 & 2. Shishikui-ikumi & Field & - \\
\hline Chondrus ocellatus & 1 & -17.0 & 8.5 & 3. Yuki & Field & - \\
\hline Gelidium elegans & 1 & -13.5 & 9.2 & 3. Yuki & Field & - \\
\hline \multicolumn{7}{|c|}{ Main Islands 'city-bay' macroalgae } \\
\hline Gelidium elegans & 1 & -14.5 & 9.6 & 4. Osaka & Stomach & 76.5 \\
\hline Chondrus giganteus & 1 & -16.6 & 9.3 & 7. Futtsu & Field & - \\
\hline Sargassum fusiforme & 1 & -14.0 & 9.7 & 7. Futtsu & Field & - \\
\hline Multiple species ${ }^{c}$ & 43 & $-16.0 \pm 2.3$ & $9.0 \pm 1.3$ & Hiroshima & Field/trawl & - \\
\hline \multicolumn{7}{|c|}{ Main Islands macroalgae (excluding Shikoku and 'city-bay' macroalgae) } \\
\hline Gelidium elegans & 1 & -17.2 & 5.3 & 5. Shimakatsu & Stomach & 40.2 \\
\hline Chondrus giganteus & 1 & -16.2 & 5.9 & 5. Shimakatsu & Stomach & 45.6 \\
\hline Codium latum & 1 & -15.1 & 5.9 & 5. Shimakatsu & Stomach & 45.6 \\
\hline Gelidium elegans & 1 & -15.9 & 6.7 & 5. Shimakatsu & Stomach & 45.6 \\
\hline Chondrus ocellatus & 1 & -19.9 & 5.0 & 5. Shimakatsu & Stomach & 46.4 \\
\hline Gelidium elegans & 1 & -16.1 & 4.4 & 6. Hachijo & Stomach & 99.0 \\
\hline Gelidium elegans & 1 & -21.1 & 5.4 & 8. Ohi & Stomach & 49.7 \\
\hline Gelidium elegans & 5 & $-16.5 \pm 1.2$ & $4.6 \pm 0.3$ & 9. Maizuru & Field & - \\
\hline Gelidium elegans & 5 & $-16.8 \pm 1.3$ & $5.6 \pm 1.6$ & 9. Maizuru & Field & - \\
\hline Gelidium linoides & 1 & -19.4 & 5.2 & 10. Tottori & Stomach & 43.7 \\
\hline Coccophora langsdorfii & 1 & -17.5 & 6.8 & 11. Okinoshima & Stomach & 71.6 \\
\hline Codium fragile & 1 & -15.8 & 5.0 & 11. Okinoshima & Stomach & 71.6 \\
\hline Prionitis angusta & 1 & -16.4 & 5.6 & 11. Okinoshima & Stomach & 71.6 \\
\hline Sargassum macrocarpum & 1 & -15.1 & 6.3 & 11. Okinoshima & Stomach & 71.6 \\
\hline Gelidium elegans & 1 & -16.3 & 7.1 & 12. Shimane & Stomach & - \\
\hline Gelidium elegans & 1 & -18.9 & 7.9 & 13. Tsuyazaki & Stomach & - \\
\hline Gelidium elegans & 1 & -15.7 & 5.3 & 14. Nomaike & Stomach & 78.7 \\
\hline \multicolumn{7}{|l|}{ Yaeyama macroalgae $^{a}$} \\
\hline Gelidiella acerosa & 1 & -13.9 & 4.6 & 15. Kuroshima & Field & - \\
\hline Gelidiella acerosa & 10 & $-15.3 \pm 1.8$ & $3.6 \pm 0.8$ & 15. Kuroshima & Field & - \\
\hline Codium sp. & 5 & $-12.4 \pm 2.2$ & $3.6 \pm 0.6$ & 15. Kuroshima & Field & - \\
\hline Codium sp. & 10 & $-12.0 \pm 1.9$ & $3.6 \pm 0.4$ & 15. Kuroshima & Field & - \\
\hline Gelidiella acerosa & 4 & $-14.3 \pm 0.1$ & $4.3 \pm 0.2$ & 15. Kuroshima & Field & - \\
\hline Betaphycus gelatinum & 4 & $-13.0 \pm 1.0$ & $3.7 \pm 0.1$ & 15. Kuroshima & Field & - \\
\hline \multicolumn{7}{|l|}{ Yaeyama seagrass $^{\mathrm{a}}$} \\
\hline Thalassia hemprichii & 5 & $-7.8 \pm 0.5$ & $3.3 \pm 1.5$ & 15. Kuroshima & Field & - \\
\hline Cymodocea serrulata & 7 & $-8.9 \pm 0.8$ & $5.1 \pm 0.7$ & 15. Kuroshima & Field & - \\
\hline Thalassia hemprichii & 5 & $-6.3 \pm 0.7$ & $2.7 \pm 0.9$ & 15. Kuroshima & Field & - \\
\hline \multicolumn{7}{|l|}{ Planktonic invertebrates } \\
\hline Lepas anatifera & 1 & - & - & 1. Muroto & Stomach & 47.4 \\
\hline Chrysaora melanaster & 1 & - & - & 5. Shimakatsu & Stomach & 46.4 \\
\hline Multiple species ${ }^{\mathrm{b}, \mathrm{d}}$ & 11 & $-18.5 \pm 1.6$ & $9.4 \pm 2.2$ & NW Pacific & Trawl & \\
\hline
\end{tabular}

${ }^{a}$ Known food (defined in 'Materials and methods'); ${ }^{b}$ Hypothetical food (defined in 'Materials and methods'); ${ }^{c}$ Data from Takai et al. (2001); ${ }^{\mathrm{d} D a t a}$ from Hatase et al. $(2002,2006)$ 
Table 3. Stable isotope ratios $\left(\delta^{13} \mathrm{C}, \delta^{15} \mathrm{~N}\right)$ of food sources listed in Table 2 were represented in mixing models by means $( \pm \mathrm{SD})$ for each component food group. n: number of samples; Y: Yaeyama; S: Shikoku

\begin{tabular}{|c|c|c|c|c|}
\hline Model components (sites) & Food taxa & $\delta^{13} \mathrm{C}(\%)$ & $\delta^{15} \mathrm{~N}(\%)$ & $\mathrm{n}$ \\
\hline \multirow[t]{2}{*}{ Macroalgae / seagrass (Y only) } & Macroalgae at $Y^{a, c}$ & $-13.5 \pm 2.1$ & $3.8 \pm 0.6$ & 34 \\
\hline & Seagrass at $Y^{a, c}$ & $-7.8 \pm 1.3$ & $3.9 \pm 1.5$ & 17 \\
\hline \multirow{3}{*}{$\begin{array}{l}\text { Known food / hypothetical animal } \\
\text { food (S \& Y) }\end{array}$} & Macroalgae at $\mathrm{S}^{\mathrm{a}, \mathrm{c}}$ & $-16.5 \pm 2.2$ & $6.5 \pm 1.4$ & 28 \\
\hline & Macroalgae \& seagrass at $\mathrm{Y}^{\mathrm{a}, \mathrm{c}}$ & $-11.6 \pm 3.3$ & $3.8 \pm 1.0$ & 51 \\
\hline & Planktonic invertebrates at remote site ${ }^{\mathrm{b}, \mathrm{e}}$ & $-18.5 \pm 1.6$ & $9.4 \pm 2.2$ & 11 \\
\hline \multirow{2}{*}{$\begin{array}{l}\text { Known food / hypothetical 'city-bay' } \\
\text { food (S only) }\end{array}$} & Macroalgae at $\mathrm{S}^{\mathrm{a}, \mathrm{c}}$ & $-16.5 \pm 2.2$ & $6.5 \pm 1.4$ & 28 \\
\hline & Macroalgae at 'city-bay' sites ${ }^{\mathrm{b}, \mathrm{c}, \mathrm{d}}$ & $-15.9 \pm 2.2$ & $9.1 \pm 1.3$ & 46 \\
\hline
\end{tabular}

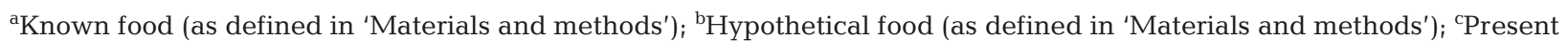
study; ${ }^{\text {T}}$ Takai et al. (2001); ${ }^{\text {Hatase et al. }(2002,2006)}$
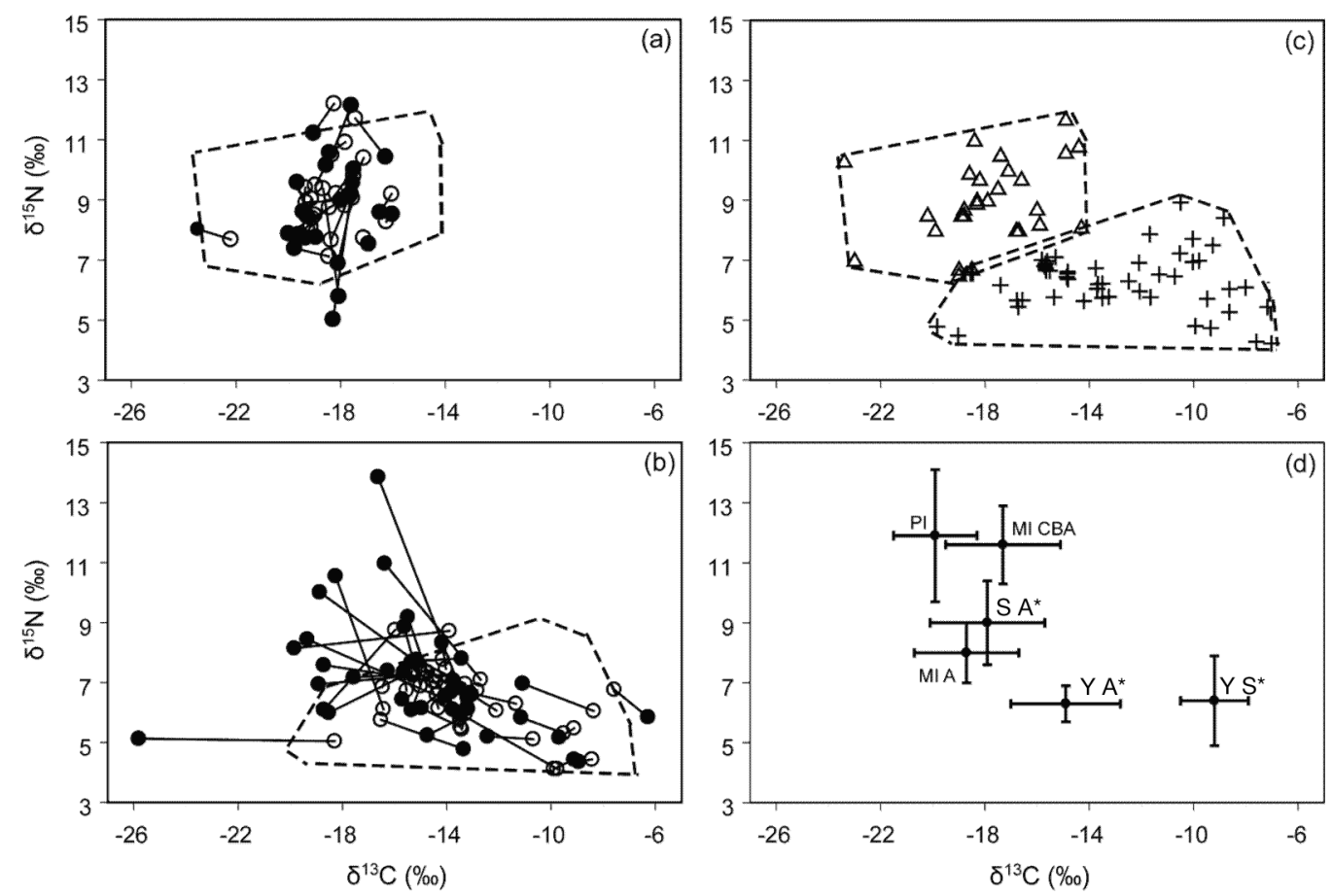

Fig. 3. Chelonia mydas. Diet history was inferred from stable isotope ratios of old scute tissue (@) and new scute tissue (O) of green turtles at (a) Shikoku $(\mathrm{n}=32)$ and $(\mathrm{b})$ Yaeyama $(\mathrm{n}=42)$. Polygons (broken line) depict isotopic boundaries for the known food (defined in 'Materials and methods') at each site. (c) Data for known food at Shikoku ( $\triangle$ ) and Yaeyama (+) in relation to isotopic boundaries. (d) Mean $( \pm \mathrm{SD}$ ) values for food sources. PI: planktonic invertebrates (hypothetical food source); MI CBA: Main Islands 'city-bay' macroalgae (hypothetical food source); S A: Shikoku macroalgae; MI A: Main Islands macroalgae, excluding Shikoku and Main Islands 'city-bay' macroalgae; Y A: Yaeyama macroalgae; Y S: Yaeyama seagrass; asterisk: 'known food' as defined in 'Materials and methods'. Food source data have been adjusted for diet-scute discrimination

of $>50 \%$ macroalgae and an estimated recent diet of $>50 \%$ seagrass. There was no apparent relationship to turtle size. Details of these model estimates can be found in Table S2 in the Supplement at www.int-res. com/articles/suppl/n025p151_supp.pdf. We caution that the biological relevance of these estimates was uncertain, as explained in the 'Discussion'.

Mixing models that included a hypothetical animal food source as well as known foods (Tables 2 \& 3) indicated that diets of Shikoku resident turtles could have included a non-trivial proportion of animal matter (mean $\pm \mathrm{SD}=39 \pm 7 \%$ ), if this was the only source additional to their known food and if the turtles had access to invertebrates with similar stable isotope values to our proxy data. The latter were sampled at a distant location as explained in 'Materials and methods'. For all Shikoku residents, the known food (macroalgae) was estimated to be the larger compo- 
nent of the diet. Similarly, for Yaeyama, the known food (macroalgae and seagrass) of all residents was estimated to be the larger component of the diet. Their estimated proportion of hypothetical animal matter (mean $\pm \mathrm{SD}=18 \pm 10 \%$ ) was smaller than that of Shikoku turtles. Details of all model estimates are available in Table S2.

As a post hoc addition explained in the 'Discussion', we ran mixing models (as detailed in 'Materials and methods') for a different hypothetical food source, together with the known food for Shikoku residents. The hypothetical food source was a subset of food source data for Main Islands sites, namely macroalgae growing in enclosed bays near large cities (Tables 2 \& 3). The model estimates indicated diets of Shikoku resident turtles could have included a non-trivial proportion of macroalgae that had stable isotope values corresponding to those of samples sourced from bays near large cities (mean $\pm \mathrm{SD}=33 \pm 11 \%$ ). Details of these model estimates are available in Table S2.

\section{DISCUSSION}

We confirmed that the known food of green turtles, as depicted by stable isotope ratios, differed significantly between our 2 study sites. Therefore, any turtles foraging at either site for extended periods could be expected to have distinctive scute isotope values that corresponded to their site. Our results indicated the majority of study turtles ( $81 \%$ at Shikoku, $64 \%$ at Yaeyama) could be considered long-term residents, having previous diets (incorporated in old scute tissue) and recent diets (incorporated in new scute tissue) corresponding to the known food at their respective sites.

Our finding of site fidelity for the majority of study turtles (those classified as resident) was consistent with evidence from a $3 \mathrm{yr}$ tagging study at Yaeyama (Kameda et al. 2013) in which green turtles were recaptured at the site up to $744 \mathrm{~d}$ after marking. Mark-recapture data were not available for Shikoku.

At Yaeyama, a substantial minority of turtles $(n=14$, $33 \%$ ) were apparent immigrants, having previous diets beyond the range of isotope ratios for known food at this site. Collectively these apparent immigrants were significantly smaller in size than residents at Yaeyama. Isotope ratios indicated that the previous diets of immigrants were broadly similar to Shikoku diets, apart from 2 exceptions discussed below. Shikoku diets could be generalised, with caution, to other Main Islands foraging sites based on similar isotopic signatures of food sources (Fig. 3, Table 2).
On the basis of isotope ratios alone, previous diets similar to Shikoku diets did not exclude the possibility of an oceanic origin for some Yaeyama immigrants. Direct comparison of isotope ratios was not feasible because data were not available for juvenile green turtles inhabiting ocean waters near Japan. However, the change in isotope ratios shown by Yaeyama immigrants matched the direction of change found at a neritic site in Australia for new recruits from the Pacific Ocean (Arthur et al. 2008), where the latter new recruits corresponded to the smallest size cohort at the neritic site. Similarly, only the smallest Yaeyama immigrants could have been new recruits of oceanic origin, although new recruit status could not be confirmed. A white plastron with prominent ridges can assist in identifying new recruits, e.g. Limpus et al. (2005), but we were unable to assess such evidence because the plastron morphology of our study turtles had not been examined in detail.

We concluded that the majority of the Yaeyama immigrants had diets consistent with a Main Islands origin and had body sizes broadly consistent with the under-represented size cohort previously reported for Main Islands sites (e.g. Okamoto et al. 2011 and references therein), and thus supported the proposition that Yaeyama immigrants could have made an ontogenetic shift from Main Islands foraging grounds. (The 'majority of immigrants' cannot be exactly enumerated because the under-represented size cohort is not bounded by exact limits, nor is there an exact size limit for potential new recruits from oceanic habitat.)

Two Yaeyama immigrants had previous diets very different from other immigrants. The first of these $(\mathrm{SCL}=58.6 \mathrm{~cm})$ had old scute data falling far to the left of the Yaeyama food boundary (Fig. 3). Erroneous data for this turtle cannot be ruled out, but the authors believe error is unlikely. The $\delta^{13} \mathrm{C}$ value $(-25.6 \%)$ suggests a diet of terrestrial plant matter. Broadly similar $\delta^{13} \mathrm{C}$ values have been found for leaves and fruits of mangroves, and these items have been confirmed as forming a part, albeit minor, of the diet eaten by green turtles in some other parts of the world (e.g. Arthur et al. 2009). We found no records of mangrove consumption by green turtles in Japan. The origins of this Yaeyama immigrant remain a mystery. The second case was a turtle of adult size $(\mathrm{SCL}=83 \mathrm{~cm}$ ) with its old scute data point to the right of the Yaeyama food boundary. From its isotope ratios, we surmise this turtle might have migrated from another sub-tropical or tropical area where seagrass was available. 
At Shikoku, the apparent immigrants were a small minority $(\mathrm{n}=4)$. Stable isotope ratios (old scute) for the 2 larger individuals $(\mathrm{SCL}=75.9$ and $63.3 \mathrm{~cm}$ ) were only slightly different to Shikoku residents, suggesting these turtles might not be immigrants and might simply have consumed other food (in modest proportion), as well as known food for Shikoku. However, the 2 smaller individuals ( $\mathrm{SCL}=37.7$ and $42.0 \mathrm{~cm}$ ) had stable isotope ratios (old scute) consistent with Yaeyama residents. The old-to-new-scute change in isotope ratios for these 2 small turtles was contrary in direction to that identified in new recruits from oceanic habitats (Arthur et al. 2008), and showed these 2 turtles had not recruited to Shikoku directly from the ocean. We surmised the 2 small immigrants at Shikoku might previously have (1) recruited from the ocean to Yaeyama (or similar Nansei Islands sites), (2) remained long enough (years) for old scute tissue to become consistent with Yaeyama food, then (3) made an ontogenetic shift to Shikoku. The latter shift would have occurred at a relatively early stage, i.e. while these 2 immigrants were still among the smallest size cohort at neritic foraging grounds in Japan (e.g. Okamoto et al. 2011 and references therein, Kameda et al. 2013).

Taking a 'big picture' view, the apparent ontogenetic movement by some Japanese green turtles from Main Islands sites towards the Nansei Islands may be in the direction of their natal origin, as has been reported for the western North Atlantic (e.g. Moncada et al. 2006, Meylan et al. 2011). The Kuroshio Current flows north-eastward (Henry \& Yoshida 1972) and may assist hatchling green turtles originating from rookeries in the Nansei Islands during their passive migration towards oceanic habitat. After their oceanic phase, if some of these turtles recruit to the Main Islands of Japan and remain at Main Islands sites during their early neritic stage, their subsequent ontogenetic shift (consistent with our original proposition and our study findings) to the southern Nansei Islands including Yaeyama would represent movement closer to their natal region. However, we remain cautious about such broad inference and suspect more complex and diverse patterns may exist. Greater insight could be gained through future use of satellite tracking, particularly for juvenile green turtles of known genetic origin.

Our mixing model estimates suggested that individual study turtles each consumed an unequal mix of macroalgae and seagrass, based on scute tissue representing food assimilated over a period of years, a duration estimated to span about 1.4 to $6.5 \mathrm{yr}$ (Fig. S1 in the Supplement at www.int-res.com/articles/suppl/ n025p151_supp.pdf and Vander Zanden et al. 2013). Interpretation was relatively clear for 8 turtles (macroalgae more important in 5 instances, seagrass more important in 3 cases) and less clear for others. We were unable to define a numeric threshold to distinguish between biologically relevant imbalance and trivial imbalance because there was no identifiable null point for comparison. Numerically equal proportions would not be expected, even if a turtle always consumed food at random. A study-relevant random selection of the 2 taxa could not be defined in the absence of detailed data for fine-scale distribution, nutritional value and abundance of these 2 taxa at the study site over the years represented by scute tissue.

Relative importance of macroalgae and seagrass in green turtle diets holds particular interest for some researchers. Digestion appears to be more efficient if a turtle consumes one or the other rather than both taxa (Bjorndal 1979, Bjorndal et al. 1991). This difference in digestive efficiency has been suggested as a possible explanation for selective foraging, the latter observed over short temporal duration at diverse locations (Bjorndal 1997 and references therein) and inferred for green turtles in Japan, similarly referring to a relatively short time span (gut content analysis by the Sea Turtle Association of Japan unpubl. data). However, assessing this taxonomic dichotomy in diet over a much longer time span through application of Bayesian mixing models proved problematic. For our mixing models, the 2 food sources were treated as equally likely to be consumed (i.e. uniform priors were specified) although it was implausible that study turtles always had equal opportunity to eat both foods. As noted above, there were no data available to support informative priors. We therefore caution that biological relevance of our proportional estimates for macroalgae and seagrass remains uncertain. The topic might be more effectively addressed for a shorter time span (provided corresponding data for food availability could be obtained) by measuring isotope ratios of tissue with relatively rapid turnover such as blood or plasma (e.g. Reich et al. 2008).

The inclusion of animal matter in our mixing models confirmed that this could be a cryptic component of green turtle diets at our study sites if the turtles had access to invertebrates with isotope values similar to the proxy data we used. The estimates indicated a greater proportion of animal matter for Shikoku diets than for Yaeyama diets. Our modelling used stable isotope ratios for known food (macroalgae and seagrass) sampled at our study sites, together with proxy values for the hypothetical animal food. The proxy data came from samples col- 
lected in Japanese waters distant from our sites (Hatase et al. 2002, 2006) and comprised salps, jellyfish and floating gastropods, all biologically plausible prey for green turtles (Kameda \& Ishihara 2009, present study). However, our direct evidence for animal consumption was extremely weak (in the stomach contents of 22 turtles we found a total of 2 small invertebrates). Therefore, we emphasise the hypothetical nature of our model estimates for animal consumption. We note also that uniform priors were problematic (as discussed above) for these models.

Mixing models are mathematically rigorous, but diets inferred from these models have limited biological relevance unless food sources are appropriately represented (Parnell et al. 2010). It is challenging to appropriately represent a hypothetical food source. In addition, the conundrum regarding uniform or informative priors (considered above) applies. Furthermore, inference may be confounded by the existence of more than one plausible diet component with coincidentally similar isotope ratios but disparate biological relevance. We discovered such a constraint on our inference.

It emerged that data we used to represent animal matter were relatively close to the isotope ratios for macroalgal samples from enclosed bays near large cities in the Main Islands. The latter samples ('citybay' macroalgae hereafter) were notably enriched in ${ }^{15} \mathrm{~N}$ relative to macroalgae from open areas of Main Islands, including Shikoku. Reasons for the difference in city-bay samples lay beyond the scope of our study, but differences in water quality associated with intensive human activity were plausible (Umezawa et al. 2002). According to mixing model estimates, if city-bay macroalgae had been consumed by Shikoku residents, then city-bay macroalgae could have comprised a slightly lower but broadly similar proportion of the diet to that estimated for animal matter.

Thus from stable isotope data we infer that diets of turtles considered resident at Shikoku could have included non-trivial quantities of animal matter, citybay macroalgae, or both. (For clarity we note that biologically relevant estimates could not be obtained from a mixing model that included 2 sources with similar stable isotope ratios.) Both hypothetical sources were plausible for Shikoku turtles. Shikoku turtles could have spent periods in offshore waters where macroplankton might be episodically abundant. Distances of 60 to $140 \mathrm{~km}$ between Shikoku and sites where city-bay macroalgae could be consumed did not preclude movement between these locations. However, no satellite tracking data were available to confirm such movement scenarios, and no compelling supportive evidence could be found in stomach samples. We recognise also that animal matter and city-bay macroalgae are not implausible in the previous diets of Yaeyama immigrants, except for the 2 individuals with exceptional diets discussed earlier. Yet the hypothetical inclusion of those sources does not contradict our original proposition regarding ontogenetic shifts from the Main Islands to the Nansei Islands.

For the green turtle foraging aggregations under consideration, known food sources and plausible hypothetical foods (when considered in biologically meaningful categories) proved to be weakly differentiated in their stable isotope values. Therefore, inference from stable isotopes must necessarily remain constrained by ambiguity, even if turtles and foods can be sampled more comprehensively in future studies. Nevertheless we conclude that the combination of stable isotope methods and SCL data have provided important support for the proposition that many juvenile green turtles make an ontogenetic shift to Yaeyama and similar Nansei Islands sites from Shikoku and similar Main Islands foraging grounds. For the majority of study turtles, stable isotope methods also indicated fidelity to Japanese foraging grounds over years. Both findings are important for future conservation management of green turtles in Japan.

Acknowledgements. Financial and logistics support were provided by STAJ, University of Tokyo and Mitsui \& Co., Ltd. Environment Fund. We thank R. Kawai, D. Oshima, H. Tanaka, T. Ishihara, K. Okamoto, Y. Ouchi, S. Yamashita, Y. Yasuoka, K. Ebisui, K. Hashimoto and Y. Kamiya; STAJ volunteers and interns; N. Akama, S. Pu, M. Sato, Y. Nakane, K. Miyauchi and Y. Takeuchi; Takaoka, Shiina and Mitsu pound net fishery associations for their help in collecting samples; H. Sugisaki, M. Kodama, K. Yamada, Y. Fukuyo and T. Masuda for their support in stable isotope analysis; and R. Jones for statistical help. Sampling permits were issued by Marine Fisheries Coordinating Committee of Okinawa prefecture (category 'Oki Cho K', No. 21-16, 22-6, 23-1), Okinawa prefecture (category 'Toku', No. 24-30) and Yaeyama Fishermen's co-operative (category 'Ya Gyo Kyo Hatsu', No. 262).

\section{LITERATURE CITED}

Amorocho DF, Reina RD (2007) Feeding ecology of the East Pacific green sea turtle Chelonia mydas agassizii at Gorgona National Park, Colombia. Endang Species Res 3:43-51

Arthur KE, O'Neil JM, Limpus CJ, Abernathy K, Marshall G (2007) Using animal-borne imaging to assess green turtle (Chelonia mydas) foraging ecology in Moreton Bay, Australia. Mar Technol Soc J 41:9-13 
Arthur KE, Boyle MC, Limpus CJ (2008) Ontogenetic changes in diet and habitat use in green sea turtle (Chelonia mydas) life history. Mar Ecol Prog Ser 362:303-311

Arthur KE, McMahon KM, Limpus CJ, Dennison WC (2009) Feeding ecology of green turtles (Chelonia mydas) from Shoalwater Bay, Australia. Mar Turtle Newsl 123:6-12

Balazs GH (1976) Green turtle migrations in the Hawaiian archipelago. Biol Conserv 9:125-140

Balazs GH (1994) Homeward bound: satellite tracking of Hawaiian green turtles from nesting beaches to foraging pastures. In: Schroeder BA, Witherington BE (comp) Proc 13th Annu Symp Sea Turtle Biol Conserv. NOAA Techn Memo NMFS-SEFSC-341, Jekyll Island, GA, p 205-208

Bass AL, Witzell WN (2000) Demographic composition of immature green turtles (Chelonia mydas) from the East Central Florida coast: evidence from mtDNA markers. Herpetologica 56:357-367

Bjorndal KA (1979) Cellulose digestion and volatile fatty acid production in the green turtle, Chelonia mydas. Comp Biochem Physiol Part A Physiol 63:127-133

Bjorndal KA (1997) Foraging ecology and nutrition of sea turtles. In: Kennish MJ, Lutz PL (eds) The biology of sea turtles. CRC Press, Boca Raton, FL, p 199-231

Bjorndal KA (1999) Priorities for research in foraging habitats. In: Eckert KL, Bjorndal KA, Abreu-Grobois FA, Donnelly $M$ (eds) Research and management techniques for the conservation of sea turtles. IUCN/SSC Marine Turtle Specialist Group Publication No. 4, MTSG, Washington, DC, p 12-14

Bjorndal KA, Bolten AB (1988) Growth rates of immature green turtles, Chelonia mydas, on feeding grounds in the southern Bahamas. Copeia 1988:555-564

Bjorndal KA, Bolten AB (1995) Developmental migrations of juvenile green turtles in the Bahamas. In: Keinath JA, Barnard DE, Musick J, Bell BA (comp) Proc 15th Annu Symp Sea Turtle Biol Conserv. NOAA Techn Memo NMFS-SEFSC-387, Hilton Head, SC, p 38-39

Bjorndal KA, Suganuma H, Bolten AB (1991) Digestive fermentation in green turtles, Chelonia mydas, feeding on algae. Bull Mar Sci 48:166-171

> Boyle MC, Limpus CJ (2008) The stomach contents of posthatchling green and loggerhead sea turtles in the southwest Pacific: an insight into habitat association. Mar Biol 155:233-241

Burkholder DA, Heithaus MR, Thomson JA, Fourqurean JW (2011) Diversity in trophic interactions of green sea turtles Chelonia mydas on a relatively pristine coastal foraging ground. Mar Ecol Prog Ser 439:277-293

Cardona L, Campos P, Levy Y, Demetropoulos A, Margaritoulis D (2010) Asynchrony between dietary and nutritional shifts during the ontogeny of green turtles (Chelonia mydas) in the Mediterranean. J Exp Mar Biol Ecol 393:83-89

- Chaloupka M, Limpus C, Miller J (2004) Green turtle somatic growth dynamics in a spatially disjunct Great Barrier Reef metapopulation. Coral Reefs 23:325-335

Chaloupka M, Bjorndal KA, Balazs GH, Bolten AB and others (2008) Encouraging outlook for recovery of a once severely exploited marine megaherbivore. Glob Ecol Biogeogr 17:297-304

> Cheng IJ (2000) Post-nesting migrations of green turtles (Chelonia mydas) at Wan-An Island, Penghu Archipelago, Taiwan. Mar Biol 137:747-754

- Epperly SP, Braun J, Allison V (1995) Sea turtles in North Carolina waters. Conserv Biol 9:384-394
Folch J, Lees M, Stanley GHS (1957) A simple method for the isolation and purification of total lipids from animal tissues. J Biol Chem 226:497-509

Forbes GA, Limpus CJ (1993) A non-lethal method for retrieving stomach contents from sea turtles. Wildl Res 20:339-343

Frazier J (2003) Prehistoric and ancient historic interactions between humans and marine turtles. In: Lutz PL, Musick JA, Wyneken J (eds) The biology of sea turtles, Vol II. CRC Press, Boca Raton, FL, p 1-38

Fry B (2006) Stable isotope ecology. Springer, New York, NY > González Carman V, Falabella V, Maxwell S, Albareda D, Campagna C, Mianzan H (2012) Revisiting the ontogenetic shift paradigm: the case of juvenile green turtles in the SW Atlantic. J Exp Mar Biol Ecol 429:64-72

González Carman V, Botto F, Gaitán E, Albareda D, Campagna C, Mianzan H (2014) A jellyfish diet for the herbivorous green turtle Chelonia mydas in the temperate SW Atlantic. Mar Biol 161:339-349

Hamabata T, Nishida S, Kamezaki N, Koike H (2009) Genetic structure of populations of the green turtle (Chelonia mydas) in Japan using mtDNA control region sequences. Bull Grad School Soc Cult Stud, Kyushu Univ 15:35-50

> Hamann M, Godfrey MH, Seminoff JA, Arthur K and others (2010) Global research priorities for sea turtles: informing management and conservation in the 21st century. Endang Species Res 11:245-269

Hatase H, Takai N, Matsuzawa Y, Sakamoto W and others (2002) Size-related differences in feeding habitat use of adult female loggerhead turtles Caretta caretta around Japan determined by stable isotope analyses and satellite telemetry. Mar Ecol Prog Ser 233:273-281

Hatase H, Sato K, Yamaguchi M, Takahashi K, Tsukamoto $\mathrm{K}$ (2006) Individual variation in feeding habitat use by adult female green sea turtles (Chelonia mydas): Are they obligately neritic herbivores? Oecologia 149: $52-64$

Heithaus MR, McLash JJ, Frid A, Dill LM, Marshall G (2002) Novel insights into green sea turtle behaviour using animal-borne video cameras. J Mar Biol Assoc UK 82: 1049-1050

Henry S, Yoshida K (1972) Kuroshio; its physical aspects. University of Tokyo Press, Tokyo

Hwang YT, Millar JS, Longstaffe FJ (2007) Do $\delta^{15} \mathrm{~N}$ and $\delta^{13} \mathrm{C}$ values of feces reflect the isotopic composition of diets in small mammals? Can J Zool 85:388-396

> Jackson JBC, Kirby MX, Berger WH, Bjorndal KA and others (2001) Historical overfishing and the recent collapse of coastal ecosystems. Science 293:629-637

Kameda K, Ishihara T (2009) Gut contents analysis of green turtles (Chelonia mydas) in Japan. Umigame Newsl 81: 17-23 (in Japanese with English Abstract)

Kameda K, Wakatuki M, Kamezaki N (2013) Population structure and growth rate of the green turtle Chelonia mydas, on feeding grounds at Kuroshima Island, of Yaeyama group, Ryukyu Archipelago. Biological Magazine Okinawa 51:93-100 (in Japanese with English abstract)

Kamezaki N (1989) The nesting sites of sea turtles in the Nansei Shoto Islands. Umigame Newsl 1:7-12 (in Japanese)

> Kittinger JN, Houtan KSV, McClenachan LE, Lawrence AL (2013) Using historical data to assess the biogeography of population recovery. Ecography 36:868-872 
Kobayashi M (2001) Annual cycle of the speckle pattern on the carapace of immature hawksbill turtles (Eretmochelys imbricata) in Cuba. Amphib-reptil 22:321-328

Lahanas PN, Bjorndal KA, Bolten AB, Encalada SE, Miyamoto MM, Valverde RA, Bowen BW (1998) Genetic composition of a green turtle (Chelonia mydas) feeding ground population: evidence for multiple origins. Mar Biol 130:345-352

Lemons G, Lewison R, Komoroske L, Gaos A and others (2011) Trophic ecology of green sea turtles in a highly urbanized bay: insights from stable isotopes and mixing models. J Exp Mar Biol Ecol 405:25-32

Limpus CJ, Miller JD, Parmenter CJ, Reimer D, McLachlan N, Webb R (1992) Migration of green (Chelonia mydas) and loggerhead (Caretta caretta) turtles to and from eastern Australian rookeries. Wildl Res 19:347-358

Limpus CJ, Couper PJ, Read MA (1994) The green turtle, Chelonia mydas, in Queensland: population structure in a warm temperature feeding area. Mem Queensl Mus 35:139-154

Limpus CJ, Limpus DJ, Arthur KE, Parmenter CJ (2005) Monitoring green turtle population dynamics in Shoalwater Bay: 2000-2004. Research Publication No. 83, Great Barrier Reef Marine Park Authority Research Publication Series, Townsville

Longin R (1971) New method of collagen extraction for radiocarbon dating. Nature 230:241-242

> Mansfield KL, Wyneken J, Porter WP, Luo J (2014) First satellite tracks of neonate sea turtles redefine the 'lost years' oceanic niche. Proc R Soc Lond B Biol Sci 281: 2020133039

- Martin TG, Chadès I, Arcese P, Marra PP, Possingham HP, Norris DR (2007) Optimal conservation of migratory species. PLoS ONE 2:e751

Mendonca MT, Ehrhart LM (1982) Activity, population size and structure of immature Chelonia mydas and Caretta caretta in Mosquito Lagoon, Florida. Copeia 1982:161-167

> Meylan PA, Meylan AB, Gray JA (2011) The ecology and migrations of sea turtles. 8. Tests of the developmental habitat hypothesis. Bull Am Mus Nat Hist 357:1-70

Miller JD (1997) Reproduction in sea turtles. In: Kennish MJ, Lutz PL (eds) The biology of sea turtles. CRC Press, Boca Raton, FL, p 51-81

Miyawaki I, Kamezaki N, Kolinski S (2000) A migration record of a green turtle from Yap Islands to Muroto, Kochi Prefecture, Japan. Umigame Newsl 45:13 (in Japanese)

MOE (Ministry of Environment of Japan) (2012) Red List, Version 4. Available at: www.biodic.go.jp/rdb/rdb_f.html (accessed 12 Jun 2013)

- Moncada F, Abreu-Grobois FA, Muhlia-Melo A, Bell C and others (2006) Movement patterns of green turtles (Chelonia mydas) in Cuba and adjacent Caribbean waters inferred from flipper tag recaptures. J Herpetol 40:22-34

Moore JW, Semmens BX (2008) Incorporating uncertainty and prior information into stable isotope mixing models. Ecol Lett 11:470-480

> Nishizawa H, Naito Y, Suganuma H, Abe O and others (2013) Composition of green turtle feeding aggregations along the Japanese archipelago: implications for changes in composition with current flow. Mar Biol 160: 2671-2685

Ogasawara Marine Center (1994) Tagging studies of sea turtles in Ogasawara Is. in the fiscal 1993. Umigame Newsl 21:3-7 (in Japanese)
Okamoto K, Ishihara T, Taniguchi M, Yamashita N, Kamezaki N (2011) Occurrence of the sea turtles at the coastal water of Kumanonada. Umigame Newsl 88:13-18 (in Japanese)

Parnell A, Jackson A (2013) SIAR: Stable Isotope Analysis in R. R package version 4.2. Available at: http://CRAN.Rproject.org/package=siar (accessed 6 Oct 2013)

Parnell AC, Inger R, Bearhop S, Jackson AL (2010) Source partitioning using stable isotopes: coping with too much variation. PLoS ONE 5:e9672

R Core Team (2013) R: a language and environment for statistical computing. R Foundation for Statistical Computing, Vienna. Available at: www.r-project.org (accessed 6 Oct 2013)

> Reich KJ, Bjorndal KA, Bolten AB (2007) The 'lost years' of green turtles: using stable isotopes to study cryptic lifestages. Biol Lett 3:712-714

Reich KJ, Bjorndal KA, Martinez del Rio C (2008) Effects of growth and tissue type on the kinetics of ${ }^{13} \mathrm{C}$ and ${ }^{15} \mathrm{~N}$ incorporation in a rapidly growing ectotherm. Oecologia 155:651-663

Sato F (1989) Abstruction of green turtles in Ogasawara Islands. Umigame Newsl 1:13-14 (in Japanese)

Seminoff JA (2004) Chelonia mydas. In: IUCN 2012 IUCN Red List of threatened species, Version 20122. Available at: www.iucnredlist.org (accessed 5 Mar 2013)

> Seminoff JA, Jones TT, Eguchi T, Jones DR, Dutton PH (2006a) Stable isotope discrimination $\left(\delta^{13} \mathrm{C}\right.$ and $\left.\delta^{15} \mathrm{~N}\right)$ between soft tissues of the green sea turtle Chelonia mydas and its diet. Mar Ecol Prog Ser 308:271-278

> Seminoff JA, Jones TT, Marshall GJ (2006b) Underwater behaviour of green turtles monitored with video-timedepth recorders: What is missing from dive profiles? Mar Ecol Prog Ser 322:269-280

Shimada T (2008) Report of preliminary research of sea turtles in Hachijo Island. Umigame Newsl 79:7-8 (in Japanese with English abstract)

Song X, Wang H, Wang W, Gu H, Chan S, Jiang H (2002) Satellite tracking of post-nesting movements of green turtles Chelonia mydas from the Gangkou Sea Turtle National Nature Reserve, China, 2001. Mar Turtle Newsl 97:8-9

Suganuma H (1998) Green turtle, Chapter 21. In: Fisheries Agency (ed) Data book on rare aquatic organisms in Japan. Japan Fisheries Resource Conservation Association, Tokyo, p 240-241 (in Japanese)

Suganuma H, Tanaka S, Inoguchi E, Narushima K (2010) Understanding from the stranded sea turtles. Aquabiology 32:407-412 (in Japanese with English abstract)

Tachikawa H (1991) Carapace length and body weight of adult green turtle in Ogasawara. Umigame Newsl 8:7-10 (in Japanese)

Tachikawa H, Sasaki A (1990) Tagging study of adult green turtle in Ogasawara Islands. Umigame Newsl 6:11-15 (in Japanese)

Takai N, Hoshika A, Tanimoto T, Mishima Y, Imamura K, Yorozu A (2001) Distribution of carbon and nitrogen stable isotope ratios in macroalgae in Hiroshima Bay. Jap J Ecol 51:177-191 (in Japanese with English Abstract)

- Uchida I (1967) On the growth of the loggerhead turtle, Caretta caretta, under rearing conditions. Bull Jpn Soc Sci Fish 33:497-507 (in Japanese with English Abstract)

Uchida I, Nishiwaki M (1982) Sea turtles in the waters adjacent to Japan. In: Bjorndal KA (ed) Biology and conservation of sea turtles. Smithsonian Institution Press, 
Washington, DC, p 317-319

Umezawa Y, Miyajima T, Yamamuro M, Kayanne H, Koike I (2002) Fine-scale mapping of land-derived nitrogen in coral reefs by $\delta^{15} \mathrm{~N}$ in macroalgae. Limnol Oceanogr 47 : 1405-1416

Vander Zanden HB, Bjorndal KA, Bolten AB (2013) Temporal consistency and individual specialization in resource use by green turtles in successive life stages. Oecologia 173:767-777

- Wallace BP, DiMatteo AD, Bolten AB, Chaloupka MY and others (2011) Global conservation priorities for marine

Editorial responsibility: Paolo Casale,

Rome, Italy turtles. PLoS ONE 6:e24510

Wyneken J, Salmon M (1992) Frenzy and postfrenzy swimming activity in loggerhead, green, and leatherback hatchling sea turtles. Copeia 1992:478-484

Yamaguchi M, Suganuma H, Narushima K (2005) Nesting status of green turtles (Chelonia mydas) in Chichijima Islands, Ogasawara in 2005 and a nesting trend over the last 27 years. Umigame Newsl 66:2-6 (in Japanese with English Abstract)

Yoshida T (1998) Marine algae of Japan. Uchida Rokakuho, Tokyo (in Japanese)

Submitted: April 22, 2013; Accepted: May 21, 2014

Proofs received from author(s): August 21, 2014 